\title{
Mn 离子对掺铈钇铝石榴石发光区范围的影响
}

\author{
赵寅生 ${ }^{(1)}$, 钟家松 ${ }^{(1)}$, 向卫东 ${ }^{(1)}$ ，华伟 ${ }^{(2)}$, 杨海龙 ${ }^{(1)}$, 王京 ${ }^{(1)}$, 蔡文 ${ }^{(1)}$ 董永军 ${ }^{(3)}$, 郡明国 ${ }^{(1)}$ \\ (1) 同济大学材料科学与工程学院, 上海 201804; \\ (2) 温州大学化学与材料工程学院, 温州 325035; \\ (3) 中国科学院上海光学与精密机械研究所, 上海 201800 \\ * 联系人, E-mail: xiangweidong001@126.com \\ 2011-02-22 收稿, 2011-06-30 接受 \\ 国家自然科学基金(60708021)、温州科技计划(G20090082)、上海市基础研究重点项目(09JC1414900)、上海市光技术专项(09DZ1142002, \\ 10DZ1140400)、浙江省重大基础自然科学基金(Z4110347)和浙江省纳米技术创新平台基金(2009R50010)资助
}

\begin{abstract}
摘要 利用碳酸氢铵溶液作为沉淀剂制备了锰、铈共掺钎铝石榴石荧光粉(YAG:Ce,Mn). 实验发现, 锰离子的掺入可以增大 YAG:Ce 荧光粉的红光发光区范围, 这主要是由于锰离子 ${ }^{2} \mathrm{E}-{ }^{4} \mathrm{~A}_{2},{ }^{5} \mathrm{E}-{ }^{5} \mathrm{~T}_{2}$ 以 及 ${ }^{1} \mathrm{~T}_{2}-{ }^{5} \mathrm{~T}_{2}$ 能级的跃迁. $\mathrm{X}$ 射线衍射(X-ray diffraction, XRD)结果表明, $\mathrm{Ce}^{3+}(1.032 \AA)$ 主要取代 $\mathrm{Y}^{3+}$ $(0.900 \AA)$ 格位, $\mathrm{Mn}^{4+}(0.538 \AA)$ 和 $\mathrm{Mn}^{3+}(0.67 \AA)$ 主要取代 $\mathrm{Al}^{3+}(0.535 \AA)$ 格位. 研究结果表明, YAG: $\mathrm{Ce}_{0.06}, \mathrm{Mn}_{0.08}$ 荧光粉的发光强度最好, 发光范围最大, 其色坐标为 $(0.233,0.194)$. 由此可见, 在 YAG:Ce荧光粉中共掺 Mn 离子可以改善白光 LED (light-emitting diodes) 中 YAG 荧光粉的发光性能.
\end{abstract}

关键词

荧光粉

LED

锰离子

YAG:Ce
铈离子掺杂钇铝石榴石 $\left(\mathrm{Y}_{3} \mathrm{~A}_{5} \mathrm{O}_{12}, \mathrm{YAG}\right)$ 材料广 泛应用于闪炼晶体材料以及 LED (light-emitting diodes) 发光苂光粉 ${ }^{[1,2]}$, 例如, 阴极射线管 (cathode-ray tubes, CRTs), 场发射显示器(field emission displays, FED), 真空苂光显示器(vacuum fluorescent displays, VFDs) 以及白光 $\operatorname{LED}^{[3 \sim 5]}$. 单掺 Ce 离子 YAG 苂光粉 可以很好地被蓝光激发, 发射出高效的黄绿光 ${ }^{[2]}$. 目 前已有研究 ${ }^{[6-9]}$ 表明, 可以在 $\mathrm{Ce}$ 离子的基础上共掺 其他稀土离子, 如 $\mathrm{Pr}, \mathrm{Sm}$ 或 $\mathrm{Tb}$ 离子的掺杂可以提高 YAG 苂光粉的发光效率, 扩展菼光粉的发射范围. 但在 YAG:Ce 菼光粉中共掺过渡元素的研究非常少.

锰元素是一种具有丰富价态的过渡元素, 研 究 ${ }^{[10]}$ 表明, Mn 离子可以作为激活元素掺人 $Y A G$ 中. 不同价态的 Mn 离子 $(2+, 3+, 4+)$ 具有不同的发光特性, 而离子价态则主要取决于材料基质的电荷补偿以及 材料的制备环境. 例如, $\mathrm{Mn}^{2+}$ 离子的发射谱范围主要 在 $400 \sim 520 \mathrm{~nm}$, 主要是由于 $\mathrm{Mn}^{2+}$ 离子在能级 ${ }^{6} \mathrm{~A}_{1}-{ }^{4} \mathrm{~A}_{1}$ 和 ${ }^{6} \mathrm{~A}_{1}-{ }^{4} \mathrm{~T}_{2}$ 之间的跃迁 ${ }^{[11]} . \mathrm{Mn}^{4+}$ 离子的激发范围在 460 480 nm 之间, 主要是由于 ${ }^{2} \mathrm{E}-{ }^{4} \mathrm{~A}_{2}$ 的能级跃迁 ${ }^{[12]}$.
此外, $\mathrm{Mn}^{3+}$ 可以取代石榴石中 $\mathrm{Al}$ 离子的八面体氧空 位, 并且通过 ${ }^{5} \mathrm{E}-{ }^{5} \mathrm{~T}_{2}$ 的能级跃迁可在红橙光区产生 发射峰 ${ }^{[13,14]}$. 目前, 尽管已经有很多关于 YAG:Ce 共 掺苂光粉的研究, 但关于 YAG:Ce,Mn 苂光粉中不同 价态锰离子发光的研究很少.

YAG 苂光粉主要采用的制备方法有固相烧 结法 ${ }^{[15]}$ 、溶胶凝胶法 ${ }^{[16]}$ 、水热法 ${ }^{[17]}$ 、热离子溅射法 ${ }^{[18]}$ 以及共沉淀法 ${ }^{[19]}$. 本文主要采用共沉淀法制备了在 蓝光激发下用于白光 LED 的 $\mathrm{Ce}, \mathrm{Mn}$ 共掺 YAG 菼 光粉.

\section{1 实验方法}

（i ) 原料制备. 氧化钇(质量分数为 $99.99 \%$ )购 自长春海普瑞有限公司, 硝酸铈(分析纯)购自上海展 云化工有限公司, $\mathrm{Al}\left(\mathrm{NO}_{3}\right)_{3} \cdot 9 \mathrm{H}_{2} \mathrm{O}$ (分析纯)购自上海 展云化工有限公司, $\mathrm{MnO}_{2}$ (质量分数为 $98 \%$ ) 购自上 海国药化学试剂有限公司.

称取一定量的高纯 $\mathrm{Y}_{2} \mathrm{O}_{3}$ 粉末和 $\mathrm{MnO}_{2}$ 粉末, 在 磁力搅拌和加热条件下, 将前者溶解在浓 $\mathrm{HNO}_{3}$ 中, 
后者溶解在浓 $\mathrm{HCl}$ 中. 称取一定量的硝酸铈和硝酸 铝, 溶解于蒸馏水中, 按照 $\mathrm{Y}_{3-x} \mathrm{Ce}_{x} \mathrm{Al}_{5} \mathrm{O}_{12} \quad(x=0.02$, $0.04,0.06,0.08,0.1)$ 以及 $\mathrm{Y}_{2.94} \mathrm{Ce}_{0.06} \mathrm{Al}_{5-y} \mathrm{Mn}_{y} \mathrm{O}_{12}$ $(y=0.02,0.04,0.06,0.08)$ 的化学计量配比将上述溶液 混合, 并且保证混合液中总离子浓度为 $0.16 \mathrm{~mol} / \mathrm{L}$.

称取适量的碳酸氢铵溶于蒸馏水中, 配成 $2 \mathrm{~mol} / \mathrm{L}$ 的碳酸氢铵溶液.

(ii) 样品制备. 在磁力摚拌和室温条件下, 向 混合好的 $300 \mathrm{~mL}$ 前驱体溶液中缓慢滴加配好的 $2 \mathrm{~mol} / \mathrm{L}$ 的碳酸氢铵溶液, 滴加速度为 $2 \mathrm{~mL} / \mathrm{min}$, 滴 定完成后继续摚拌 $1 \mathrm{~h}$. 室温条件下陈化 $4 \mathrm{~h}$ 后, 进行 真空抽滤, 洗涤(蒸馏水洗 3 遍, 乙醇洗 3 遍). 沉淀 物在 $110^{\circ} \mathrm{C}$ 条件下干燥 $10 \mathrm{~h}$, 之后在 $1200^{\circ} \mathrm{C}$ 空气条件 下煅烧 $2 \mathrm{~h}$, 制成苂光粉粉末.

(iii) 样品测试. 采用 X 射线衍射仪(X-ray diffraction, XRD; Bruker D8, 德国)进行 XRD 检测, 铜 靶, $\lambda=0.15406 \mathrm{~nm}$, 加速电压为 $40 \mathrm{kV}$, 扫描范围为 $10^{\circ} \sim 70^{\circ}$, 扫描步长为 $0.02^{\circ}$. 用场发射扫描电子显微 镜 (field-emission scanning electron microscopy, FESEM; JEOL-6700F, 日本)对样品的形貌进行表征, 加速电压为 $10 \mathrm{kV}$. 采用 FluoroMax-4 光谱仪 (HORIBA Jobin Yvon 公司, 法国)进行光致激发和发 射光谱测试, 探测波长范围为 $200 \sim 750 \mathrm{~nm}$. 采用远 方 PMS-50 系统 (上海半导体照明工程技术研究中心) 进行电光源测试, 扫描范围为 380 800 $\mathrm{nm}$, 扫描间 隔为 $5 \mathrm{~nm}$. 所有测试均在室温下进行.

\section{2 结果与讨论}

\subsection{YAG:Ce 菼光粉的发光图谱}

不同 $\mathrm{Ce}$ 离子掺杂浓度的 $\mathrm{YAG}$ 苂光粉的发射光 谱和激发光谱分别如图 1(a)和(b)所示, 掺杂浓度按 照 $\mathrm{Y}_{3-x} \mathrm{Ce}_{x} \mathrm{Al}_{5} \mathrm{O}_{12}(x=0.02,0.04,0.06,0.08,0.1)$ 化学计 量比计算. 随着 $\mathrm{Ce}$ 离子浓度的不断增加, 受激发射 强度和激发强度的变化趋势基本相同. 尽管 $\mathrm{Ce}$ 离子 的掺杂浓度有所不同, 5 组苂光粉在 $460 \mathrm{~nm}$ 蓝光激发 下的最强受激发射峰都在 $528 \mathrm{~nm}$, 这主要是由于 $\mathrm{Ce}^{3+}$ 离子 ${ }^{2} \mathrm{~T}_{2 \mathrm{~g}}(5 \mathrm{~d})-{ }^{2} \mathrm{~F}_{7 / 2},{ }^{2} \mathrm{~F}_{5 / 2}(4 \mathrm{f})$ 的能级跃迁. 苂光粉 YAG:Ce $e_{0.06}, Y A G: \mathrm{Ce}_{0.08}$ 以及 YAG:Ce $\mathrm{Ce}_{0.1}$ 的发射强度和 激发强度非常相似, 其中 YAG: $\mathrm{Ce}_{0.06}$ 的受激发射峰 强度最大, 如图 1(a)所示. 这主要是由于稀土离子掺 杂达到一定量后, 会出现浓度猝灭效应 ${ }^{[20]}$.
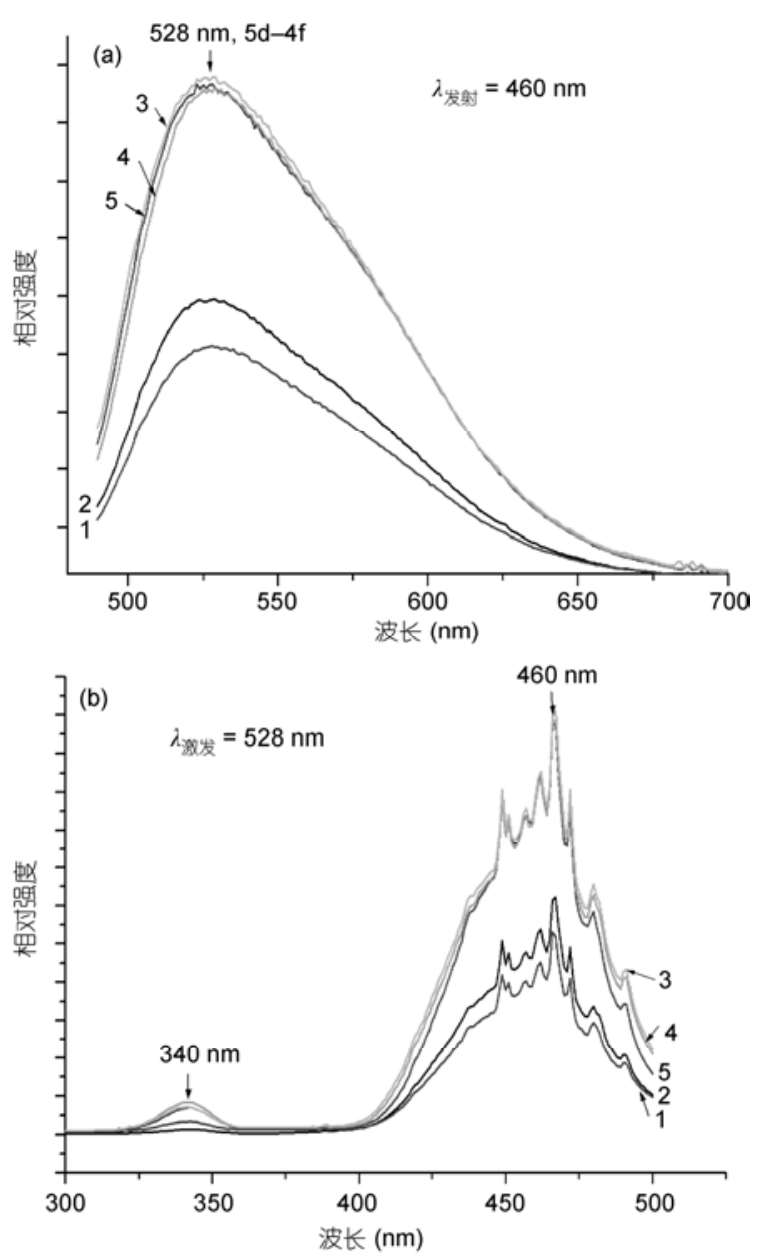

图 1 (a) YAG:Ce 荧光粉的发射图谱; (b) YAG:Ce 荧光粉的 激发图谱

1, YAG:Ce $\mathrm{C}_{0.02} ; 2$, YAG:Ce $\mathrm{C}_{0.04} ; 3, \mathrm{YAG}: \mathrm{Ce}_{0.06} ; 4, \mathrm{YAG}_{\mathrm{C}} \mathrm{Ce}_{0.08}$; 5, YAG:Ce $\mathrm{C}_{0.1}$

\section{2 苂光粉的晶型及形貌}

不同掺杂浓度的 YAG:Ce 苂光粉的发射峰相对 强度如图 1(a)所示, YAG:Ce $e_{0.06}$ 苂光粉的发射强度比 其他组分 YAG: $\mathrm{Ce}_{x}(x=0.02,0.04,0.08,0.1)$ 更强. 所

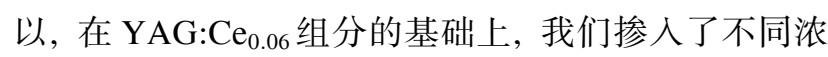
度的 $\mathrm{Mn}$ 离子. $\mathrm{YAG}: \mathrm{Ce}_{0.06}$ 苂光粉以及不同 $\mathrm{Mn}$ 离子 掺杂浓度 YAG: $\mathrm{Ce}_{0.06}, \mathrm{Mn}_{y}(y=0.02,0.04,0.06,0.08)$ 苂 光粉的 XRD 图谱如图 2 所示, 可见较强的 $\mathrm{YAG}$ 基质 相的特征衍射峰, 且峰值较高. 与标准图谱 JCPDS 卡片(33-0040)对比发现, 测试结果中无其他杂峰出 现, 说明 YAG: $\mathrm{Ce}_{0.06}, \mathrm{Mn}_{y}(y=0.02,0.04,0.06,0.08)$ 苂 光粉中 YAG 相的结晶度较高、物相较纯净. XRD 结 果表明, Mn 离子的掺人对 $\mathrm{YAG}$ 基质晶格的影响不大, 掺杂的 $\mathrm{Mn}$ 离子基本进人 $\mathrm{YAG}$ 晶格点位中. 与标准 


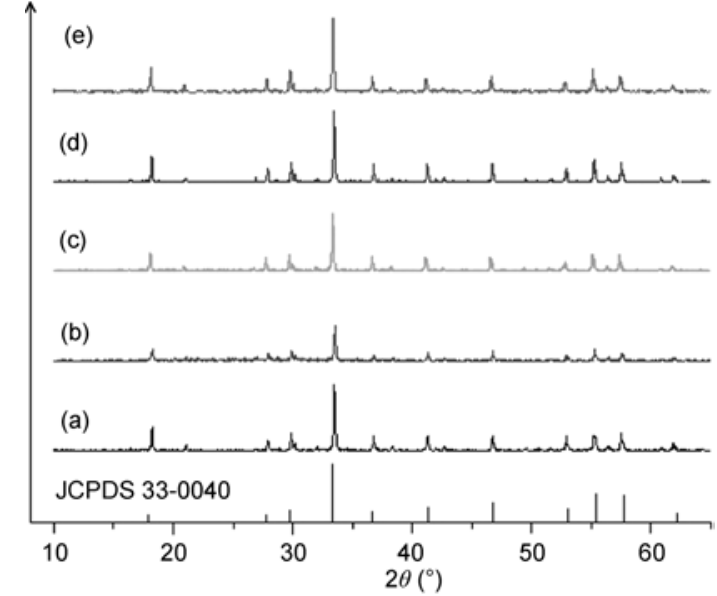

图 2 (a) YAG:Ce ${ }_{0.06}$, (b) YAG:Ce ${ }_{0.06}, \mathrm{Mn}_{0.02}$, (c) YAG:Ce $\mathrm{C}_{0.06}$, $\mathrm{Mn}_{0.04}$, (d) YAG:Ce $\mathrm{C}_{0.06}, \mathrm{Mn}_{0.06}$, (e) YAG:Ce $\mathrm{Ce}_{0.06}, \mathrm{Mn}_{0.08}$ 荧光粉 的 XRD 图谱

图谱相比, YAG:Ce $\mathrm{Ce}_{0.06}, \mathrm{Mn}_{y}(y=0.02,0.04,0.06,0.08)$ 的 $2 \theta$ 值明显向小角度偏移(图 2(b) (e)). 有研究 ${ }^{[12]}$ 表明, 这种小角度偏移是由于大尺寸掺杂离子取代了较小 的晶格点位. 由于样品的制备环境是在空气气氛中, $\mathrm{Mn}$ 离子掺杂价态主要为 +3 和 +4 价 ${ }^{[1]}$, 其中 $\mathrm{Mn}^{3+}$ 离
子半径为 $0.645 \AA, \mathrm{Mn}^{4+}$ 离子半径为 $0.538 \AA$, 而 $\mathrm{Y}^{3+}$ 离子半径为 $0.900 \AA \mathrm{Al}^{3+}$ 离子半径为 $0.535 \AA$. 由此 可见, $\mathrm{Mn}^{3+}$ 和 $\mathrm{Mn}^{4+}$ 离子主要取代 $\mathrm{YAG}$ 基质中的 $\mathrm{Al}$ 离子格位.

YAG:Ce $\mathrm{C}_{0.06}$ 和 YAG: $\mathrm{Ce}_{0.06}, \mathrm{Mn}_{0.08}$ 苂光粉在低倍放 大条件下的 SEM 图分别如图 3(a)和(b)所示, 苂光粉 的尺寸在 200 500 nm 范围内, 呈圆球状, 不同掺杂 浓度的样品形貌很相似. YAG: $\mathrm{Ce}_{0.06}, \mathrm{Mn}_{0.08}$ 苂光粉的 高倍放大 SEM 图如图 3(c)所示.

\subsection{YAG:Ce,Mn 菼光粉的发光图谱}

$\mathrm{Mn}^{3+}$ 离子的特征激发波长为 $390 \mathrm{~nm}^{[21]}$, 而 $\mathrm{Mn}^{4+}$ 和 $\mathrm{Ce}^{3+}$ 离子的特征激发波长为 $460 \mathrm{~nm}^{[12]} . \mathrm{Mn}, \mathrm{Ce}$ 共 掺 YAG 苂光粉在 $460 \mathrm{~nm}$ 波长激发下的发射图谱如 图 4 所示, 不同 $\mathrm{Mn}$ 离子掺杂浓度的 YAG: $\mathrm{Ce}$ 苂光粉 均在可见光区产生发射峰, 发射主峰值为 $538 \mathrm{~nm}$. 与 YAG:Ce 菼光粉的发射峰(图 1(a))相比, YAG:Ce, $\mathrm{Mn}$ 苂光粉在 504, 625 和 $691 \mathrm{~nm}$ 可见特有的尖锐发 射峰. 如图 4(a)所示, 随着 Mn 离子掺人量的增大, 发射峰的发射峰最强. 图 4(b)为 YAG: $\mathrm{Ce}_{0.06}, \mathrm{Mn}_{0.06}$ 苂
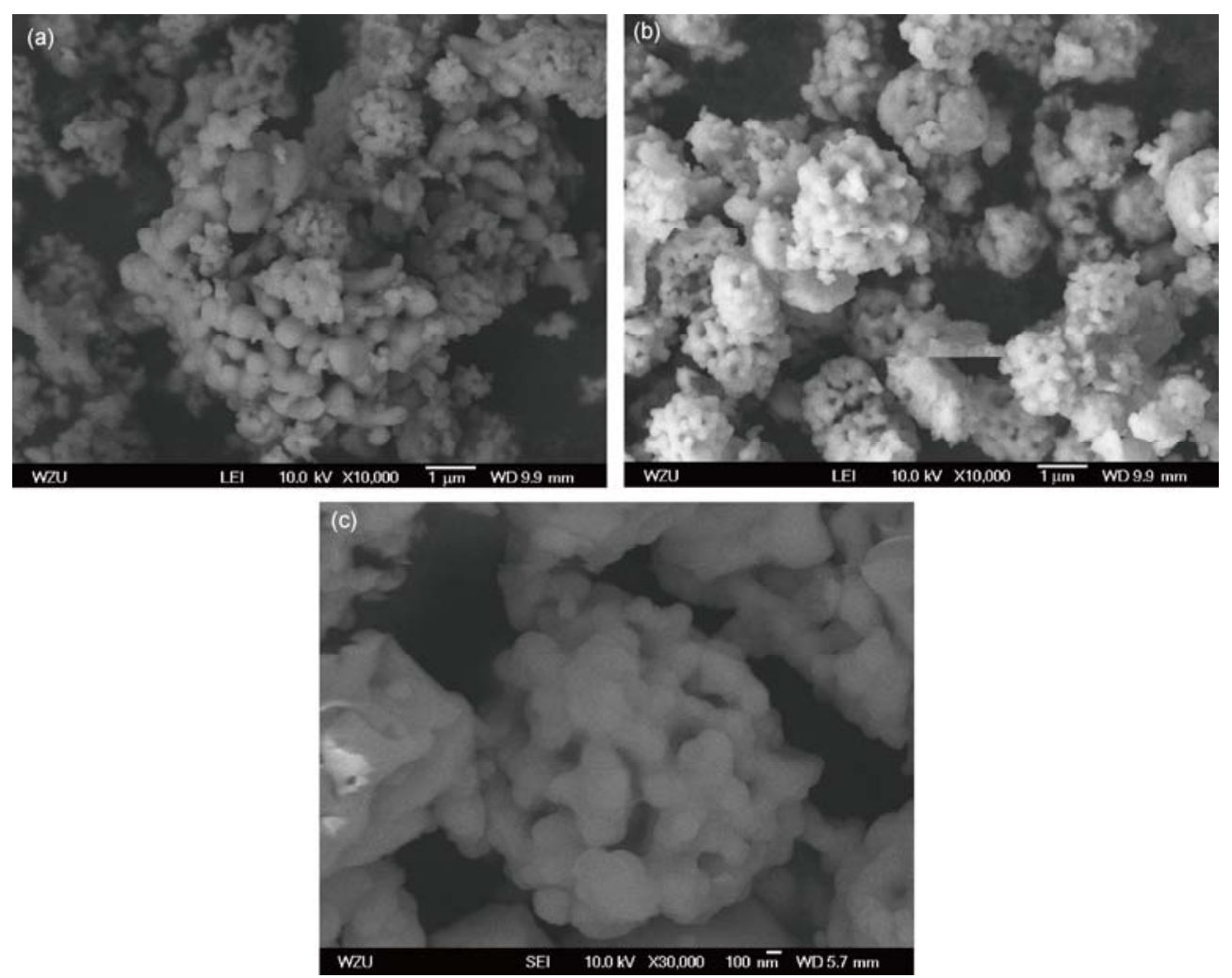

图 3 (a) YAG:Ce $\mathrm{Ce}_{0.06}$ 和 (b), (c) YAG:Ce $\mathrm{Ce}_{0.06}, \mathrm{Mn}_{0.08}$ 荧光粉的 $\mathrm{SEM}$ 图 

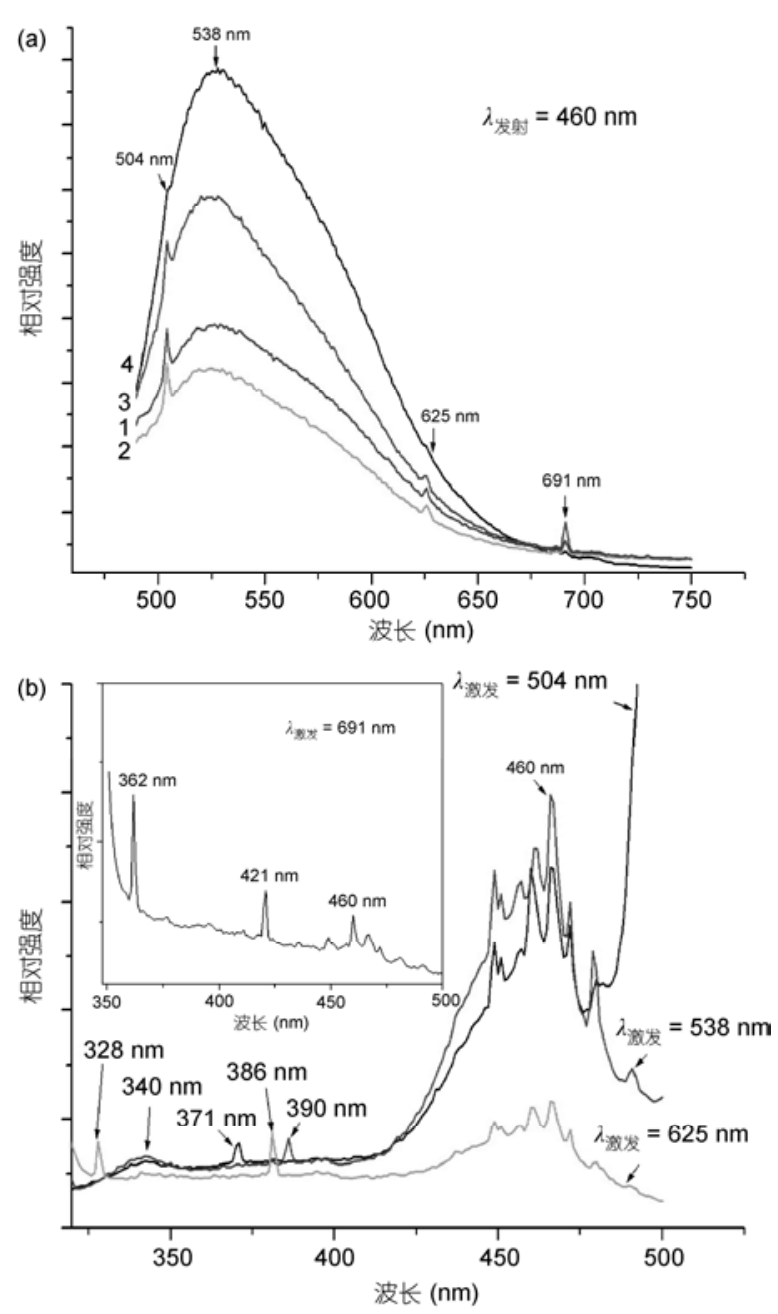

图 4 (a) YAG:Ce,Mn 荧光粉在 $460 \mathrm{~nm}$ 波长激发下的发射图 谱; (b) YAG: $\mathrm{Ce}_{0.06}, \mathrm{Mn}_{0.06}$ 荧光粉的激发图谱

1, YAG:Ce $\mathrm{Ce}_{0.06}, \mathrm{Mn}_{0.02} ; 2$, YAG:Ce $\mathrm{C}_{0.06}, \mathrm{Mn}_{0.04} ; 3, \mathrm{YAG}: \mathrm{Ce}_{0.06}, \mathrm{Mn}_{0.06}$; 4, YAG:Ce $\mathrm{C}_{0.06}, \mathrm{Mn}_{0.08}$

光粉在 504, 538, 625 和 $691 \mathrm{~nm}$ 监测波长下的激发光 谱, 激发峰范围为 320 490 nm, 说明 YAG:Ce,Mn 苂光粉可以被蓝光激发. 如图 5 所示, $\mathrm{Mn}$ 离子的掺人 使得 YAG:Ce 苂光粉的发射峰从 528 红移至 $538 \mathrm{~nm}$, 半高峰宽也扩大了 $11 \mathrm{~nm}$, 这主要是由于 $\mathrm{Mn}$ 离子 ${ }^{2} \mathrm{E}-{ }^{4} \mathrm{~A}_{2},{ }^{5} \mathrm{E}-{ }^{5} \mathrm{~T}_{2}$ 以及 ${ }^{1} \mathrm{~T}_{2}-{ }^{5} \mathrm{~T}_{2}$ 的能级跃迁 ${ }^{[12]}$. 图 6(a)为 苂光粉 YAG:Ce, $\mathrm{Mn}$ 在 $\mathrm{Mn}^{3+}$ 特征激发波长 $390 \mathrm{~nm}$ 激发下的发射图, 受激发射峰主要在 531, 641 和 744 $\mathrm{nm}$. 图 6(b) 为 $Y A G: \mathrm{Ce}_{0.06}, \mathrm{Mn}_{0.06}$ 苂光粉在 531, 641 和 $744 \mathrm{~nm}$ 波长监测下的激发谱图, 激发波长范围为 336 490 nm. 这说明 $\mathrm{Mn}$ 离子的掺人可以扩大 YAG:Ce 苂光粉的发射峰范围, 并且发射峰相对强度 有所增大.

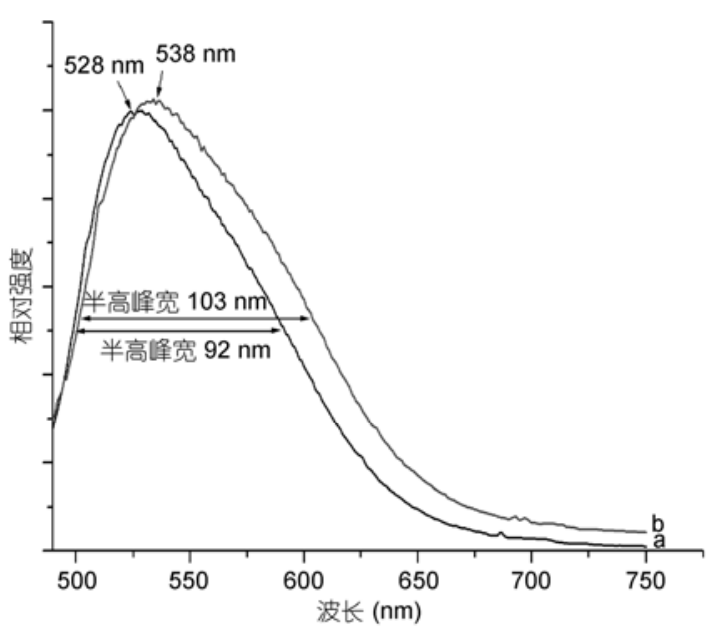

图 5 (a) YAG:Ce $\mathrm{C}_{0.06}$ 和(b) YAG: $\mathrm{Ce}_{0.06}, \mathrm{Mn}_{0.08}$ 荧光粉的半高 峰宽和激发强度对比图
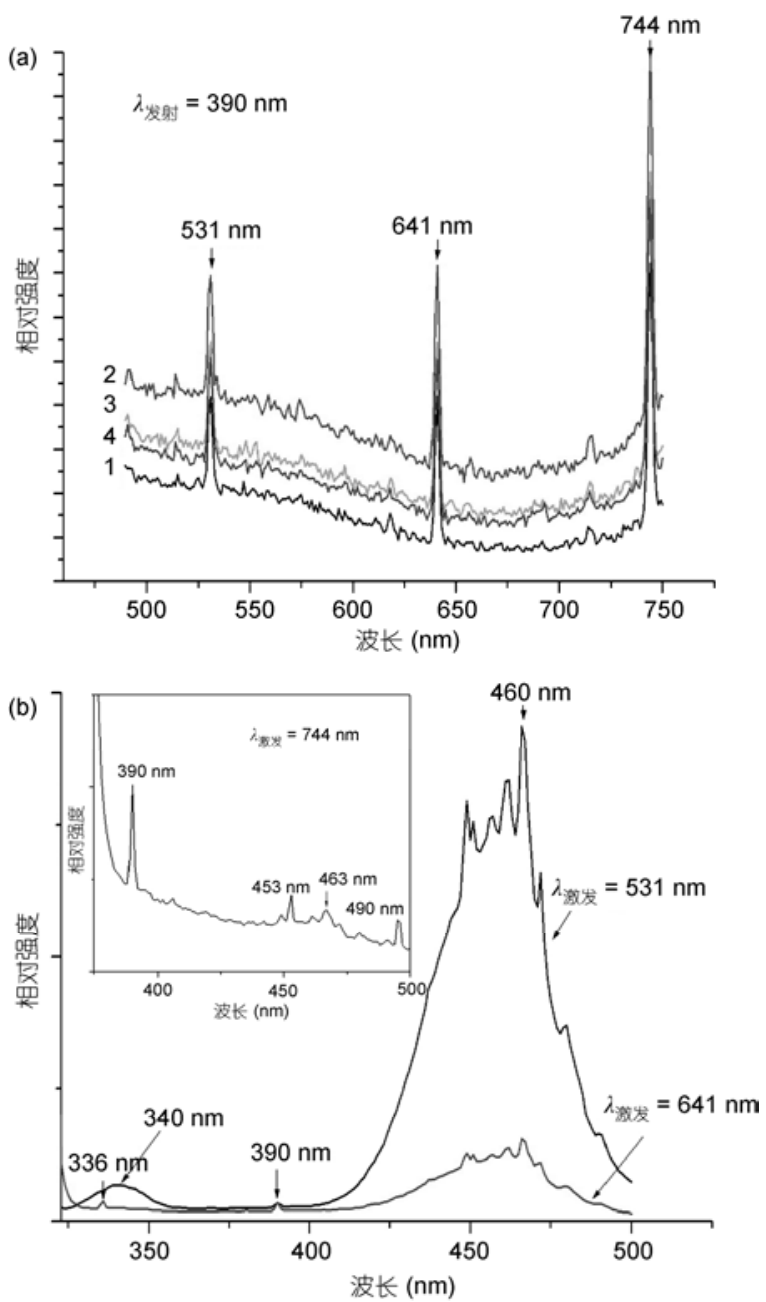

图 6 (a) YAG:Ce,Mn 荧光粉在 $390 \mathrm{~nm}$ 波长激发下的发射图 谱; (b) YAG: $\mathrm{Ce}_{0.06}, \mathrm{Mn}_{0.06}$ 苂光粉的激发图谱

1, YAG: $\mathrm{Ce}_{0.06}, \mathrm{Mn}_{0.02} ; 2$, YAG: $\mathrm{Ce}_{0.06}, \mathrm{Mn}_{0.04} ; 3$, YAG: $\mathrm{Ce}_{0.06}, \mathrm{Mn}_{0.06}$; 4, YAG: $\mathrm{Ce}_{0.06}, \mathrm{Mn}_{0.08}$ 


\section{4 苂光粉的光电性能}

表 1 为不同掺杂浓度的 YAG:Ce,Mn 苂光粉在 $460 \mathrm{~nm}$ 蓝光波长监测下的光电参数. 通过色坐标参 数可见, 随着 $\mathrm{Mn}$ 离子掺人量的增大, YAG:Ce,Mn 菼 光粉比 YAG:Ce 菼光粉更靠近白光发光区. 图 7 为 YAG: $\mathrm{Ce}_{0.06}, \mathrm{YAG}: \mathrm{Ce}_{0.06}, \mathrm{Mn}_{0.04}, \mathrm{YAG}: \mathrm{Ce}_{0.06}, \mathrm{Mn}_{0.08}$ 苂 光粉的 CIE 坐标图, 其中 YAG: $\mathrm{Ce}_{0.06}$ 苂光粉的 CIE 色坐标为 $(0.203,0.167)$, 相比而言, $\mathrm{YAG}: \mathrm{Ce}_{0.06}, \mathrm{Mn}_{0.04}$, YAG: $\mathrm{Ce}_{0.06}, \mathrm{Mn}_{0.08}$ 的 CIE 色坐标更接近白光区, 分别 为 $(0.249,0.181)$ 和 $(0.233,0.194)$.

\section{3 结论}

研究发现, Mn 离子的掺人可以使 YAG:Ce 苂光 粉的发光峰红移 $10 \mathrm{~nm}$ 左右, 半高峰宽扩大 $11 \mathrm{~nm}$, 这主要是由于不同价态 $\mathrm{Mn}$ 离子 ${ }^{2} \mathrm{E}-{ }^{4} \mathrm{~A}_{2},{ }^{5} \mathrm{E}-{ }^{5} \mathrm{~T}_{2}$ 以及 ${ }^{1} \mathrm{~T}_{2}-{ }^{5} \mathrm{~T}_{2}$ 能级的跃迁. 随着 $\mathrm{Mn}$ 离子掺人量的增大, YAG:Ce,Mn 菼光粉的发射强度也不断增大. 实验表 明, YAG: $\mathrm{Ce}_{0.06}, \mathrm{Mn}_{0.08}$ 菼光粉的发光强度最好, 发光 范围最宽, 其色坐标为 $(0.233,0.194)$. 由此可见, YAG:Ce 苂光粉中共掺 Mn 离子可以改善白光 LED 中 YAG 苂光粉的发光性能.
表 1 荧光粉 YAG: $\mathrm{Ce}_{0.06}, \mathrm{Mn}_{y}$ 在 $460 \mathrm{~nm}$ 监测下的光电参数

\begin{tabular}{ccc}
\hline $\mathrm{Mn}$ 离子浓度 $(\mathrm{mol} / \mathrm{L})$ & $\mathrm{CIE} x$ 值 & $\mathrm{CIE} y$ 值 \\
\hline 0 & 0.203 & 0.167 \\
0.02 & 0.207 & 0.157 \\
0.04 & 0.249 & 0.181 \\
0.06 & 0.22 & 0.173 \\
0.08 & 0.233 & 0.194 \\
\hline
\end{tabular}

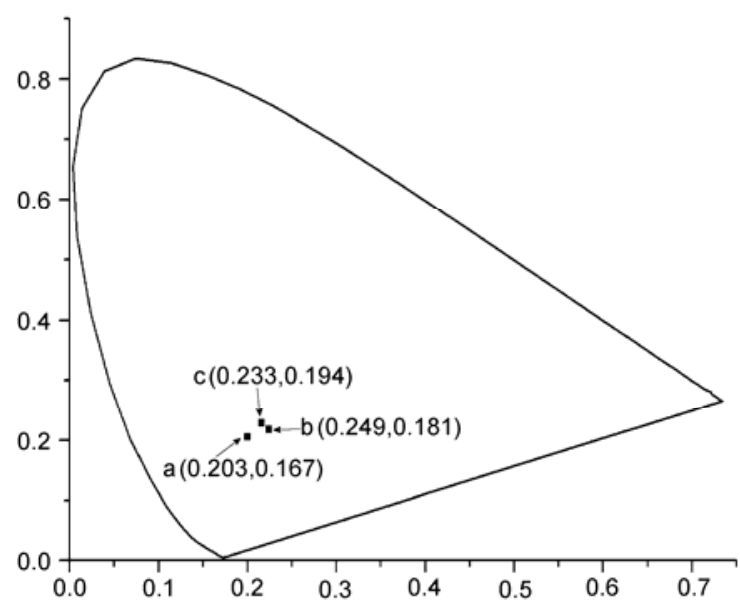

图 7 不同荧光粉的 CIE 坐标图

a, YAG:Ce $e_{0.06} ;$ b, YAG:Ce $e_{0.06}, \mathrm{Mn}_{0.04} ; \mathrm{c}, \mathrm{YAG}: \mathrm{Ce}_{0.06}, \mathrm{Mn}_{0.08}$

\section{参考文献}

1 Hassanzadeh-Tabrizi S A, Taheri-Nassaj E, Sarpoolaky H. Synthesis of an alumina-YAG nanopowder via sol-gel method. J Alloy Compd, 2008, 456: 282-285

2 Kottaisamy M, Thiyagarajan P, Mishra J, et al. Color tuning of $\mathrm{Y}_{3} \mathrm{Al}_{5} \mathrm{O}_{12}$ :Ce phosphor and their blend for white LEDs. Mater Res Bull, 2008, 43: 1657-1663

3 Zhang K, Liu H Z, Wu Y T, et al. Co-precipitation synthesis and luminescence behavior of Ce-doped yttrium aluminum garnet (YAG:Ce) phosphor: The effect of precipitant. J Alloy Compd, 2008, 453: 265-270

4 Zhang N, Wang D J, Li L. YAG:Ce phosphors for WLED via nano-pesudoboehmite sol-gel route. J Rare Earth, 2006, 24: 294-297

5 Wu Z G, Zhang X D, He W. Solvothermal synthesis of spherical YAG powders via different precipitants. J Alloy Compd, 2009, 472: $576-580$

6 Yang H S, Kim Y S. Energy transfer-based spectral properties of Tb-, Pr-, or Sm-codoped YAG:Ce nanocrystalline phosphors. J Lumin, 2008, 128: 1570-1576

7 Zhou Y, Lin J, Yu M, et al. Synthesis-dependent luminescence properties of $\mathrm{Y}_{3} \mathrm{Al}_{5} \mathrm{O}_{12}: R e^{3+}(R e=\mathrm{Ce}, \mathrm{Sm}, \mathrm{Tb})$ phosphors. Mater Lett, 2002, 56: 628-636

8 Ho S J, Won B L, Dong C L, et al. Enhancement of red spectral emission intensity of $\mathrm{Y}_{3} \mathrm{Al}_{5} \mathrm{O}_{12}: \mathrm{Ce}^{3+}$ phosphor via Pr co-doping and $\mathrm{Tb}$ substitution for the application to white LEDs. J Lumin, 2007, 126: 371-377

9 Nakielska M, Kosko J, Sarnecki J, et al. Fluorescence properties in the visible of highly $\mathrm{Pr}^{3+}$ doped YAG planar waveguides. Opt Mater, 2008, 30: 759-762

10 Noginov M A, Loutts G B, Warren M. Spectroscopic studies of $\mathrm{Mn}^{3+}$ and $\mathrm{Mn}^{2+}$ ions in $\mathrm{YAlO}_{3}$. J Opt Soc Am B, 1999, 16: 475-483

11 Yu Z, Gorbenko V, Voznyak T, et al. Luminescence of $\mathrm{Mn}^{2+}$ ions in $\mathrm{Tb}_{3} \mathrm{Al}_{5} \mathrm{O}_{12}$ garnet. J Lumin, 2010, 130: 380-386

12 Donegan J F, Glynn T J, Imbusch G F. Luminescence and fluorescence line narrowing studies of $\mathrm{Y}_{3} \mathrm{Al}_{5} \mathrm{O}_{12}: \mathrm{Mn}^{4+}$. J Lumin, 1986, 36: 93-100 
13 Stefan K, Simone H, Stephan H, et al. $\mathrm{Mn}^{3+}$ fundamental spectroscopy and excited state absorption. Phys Solid State, 1998, 8: 206-209

14 Stefan K, Simone H, Stephan H, et al. Emission of octahedrally coordinated $\mathrm{Mn}^{3+}$ in garnets. Spectrochim Acta A, 1998, 54: 1741-1749

15 Stefan K, Simone H, Stephan H, et al. Optical transition in $\mathrm{Mn}^{3+}$ doped garnets. Phys Rev B, 1998, 57: 2203-2216

16 Kyung N K, Jung H K, Hee D P, et al. High luminance of new green emitting phosphor $\mathrm{Mg}_{2} \mathrm{SnO}_{4}:$ Mn. J Lumin, 2002, 99: 169-173

17 Kasuya R, Isobe T, Kuma H. Glycothermal synthesis and photoluminescence of YAG:Ce ${ }^{3+}$ nanophosphors. J Alloy Compd, 2006, 408-412: $820-823$

18 Julien M, Tyrone J, Rita B, et al. Yttrium aluminum garnet nanopowders produced by liquid-feed flame spray pyrolysis of metallooraganic precursors. Chem Mater, 2004, 16: 822-831

19 Yuan F L, Ryu H J. Ce-doped YAG phosphor powders prepared by co-precipitation and heterogeneous precipitation. Mater Sci Eng B, 2004, 107: 14-18

20 Pan Y X, Wu M M, Su Q. Tailored photoluminescence of YAG:Ce phosphor through various methods. J Phys Chem Solids, 2004, 65: $845-850$

21 Klaus P, Gunter H. Broad band fluorescence of transition metal doped garnets and tungstates. J Lumin, 1984, 31-32: 71-77 